\title{
ESTUDO DA AUDIÇÃO E DA QUALIDADE DE VIDA EM MOTORISTAS DE CAMINHÃO
}

\section{Study of hearing and quality of life in truck drivers}

\author{
Gabriela Lopes ${ }^{(1)}$, lêda Chaves Pacheco Russo ${ }^{(2)}$, Ana Cláudia Fiorini ${ }^{(3)}$
}

\section{RESUMO}

Objetivo: estudar a audição e a sua relação com a qualidade de vida em motoristas de caminhão. Métodos: realizou-se um estudo epidemiológico do tipo transversal de inquérito. Estudou-se 75 motoristas de caminhão, que responderam a anamnese, preencheram o questionário sobre Qualidade de vida (WHOQOL-bref) e se submeteram a audiometria tonal liminar e logoaudiometria. Classificou-se a Perda Auditiva Induzida por Ruído (PAIR), segundo o critério utilizado por Fiorini (1994). Resultados: dos 75 audiogramas analisados, 50 sujeitos (66,7\%) apresentaram audição dentro dos padrões de normalidade (Grupo 1) e 25 sujeitos (33,3\%) apresentaram audiogramas com alterações: $20(80,0 \%)$ foram classificados como sugestivos de PAIR (Grupo 2) e cinco (20,0\%) foram sugestivos de outras causas (Grupo 3). Houve associação e/ou dependência entre os Grupos e a Idade $(p=0,007)$ e entre os Grupos e os Anos de Profissão ( $p=0,049)$. No Grupo 1 - Normalidade, houve correlações estatisticamente significantes para os domínios: Físico x Psicológico $(p<0,001)$, Social x Físico $(p<0,001)$, Meio Ambiente x Físico $(p<0,001)$, Psicológico $\times$ Social $(p=0,002)$, Psicológico x Meio Ambiente $(p=0,001)$ e Meio Ambiente $x$ Social $(p<0,001)$. No Grupo 2 - Sugestivo de PAIR, as correlações foram: Físico $x$ Psicológico $(p=0,002)$, Psicológico $x$ Social $(p<0,001)$, Psicológico $x$ Ambiente $(p=0,005)$ e Ambiente $x$ Social $p=(0,026)$. Conclusão: a prevalência de alterações auditivas sugestivas de PAIR foi de $28,6 \%$ nesta classe profissional, porém, a qualidade de vida não esteve relacionada aos resultados obtidos nos audiogramas.

DESCRITORES: Perda Auditiva; Qualidade de Vida; Saúde do Trabalhador; Ruído Ocupacional; Audiometria; Audiologia

\section{INTRODUÇÃO}

O transporte rodoviário é o meio mais importante para suprir as necessidades dos brasileiros, movimentando a economia em todo o território nacional. O elevado valor do veículo, as vidas sob sua responsabilidade, as cargas valiosas e tóxicas, a sinalização deficiente das vias e estradas igualmente defeituosas, caracterizam desafios que o motorista

(1) Fonoaudióloga; Support - Assessoria em Fonoaudiologia; Mestre em Fonoaudiologia pela Pontifícia Universidade de São Paulo.

(2) Fonoaudióloga; Professora Titular do Programa de Estudos Pós-Graduados em Fonoaudiologia da Pontifícia Universidade Católica de São Paulo; Doutora em Distúrbios da Comunicação Humana pela Universidade Federal de São Paulo.

(3) Fonoaudióloga; Professora Associada do Programa de Estudos Pós-Graduados em Fonoaudiologia da Pontifícia Universidade Católica de São Paulo; Doutora em Saúde Pública pela Faculdade de Saúde Pública da Universidade de São Paulo. de caminhão é obrigado a enfrentar em sua jornada de trabalho. $O$ excesso de ruído, o calor que desprende de dentro da cabine, a conformação da poltrona, nem sempre anatomicamente correta, e a permanência em uma mesma posição em frente ao volante, demandam desses profissionais uma intensa atividade física e mental ${ }^{1}$.

No Canadá, foram realizadas mais de 400 medições, para avaliar o ruído a que os motoristas de caminhão estavam expostos, em oito horas de trabalho. Observou-se que, dirigindo com as janelas fechadas e rádio desligado, foram encontrados os mais reduzidos níveis sonoros de exposição ao ruído. O Nível Sonoro Equivalente (Leq) encontrado nos motoristas durante suas atividades foi de 78 a $89 \mathrm{~dB}(\mathrm{~A})$ com um valor médio de $82,7 \mathrm{~dB}(\mathrm{~A})$ e dirigindo com a janela aberta e com o rádio ligado, resultou em um aumento do valor médio em 3,9 dB (A). Portanto, a exposição excedeu o valor limite permitido por lei para garantir a saúde auditiva, ou seja, $85 \mathrm{~dB}(\mathrm{~A})$ por oito horas ${ }^{2}$. 
No Brasil, a ocorrência de perda auditiva foi estudada em 111 motoristas de ônibus e 157 de caminhão com, no mínimo, dois anos de exposição ao ruído e à vibração de corpo inteiro, no período de três anos. Os resultados apontaram uma prevalência de 4,5\% e 11,5\% de audiogramas sugestivos de Perda Auditiva Induzida por Ruído (PAIR) em motoristas de ônibus (Grupo I) e caminhões (Grupo II), respectivamente. A dose de exposição diária ao ruído para o Grupo I foi de $80,3 \mathrm{~dB}(\mathrm{~A})$ e para o Grupo II foi de $87 \mathrm{~dB}(\mathrm{~A})^{3}$.

A saúde auditiva dos motoristas de caminhão ainda não foi valorizada e insistentemente explorada pelos pesquisadores. Ao se preocuparem com esta questão, diversos trabalhos internacionais apontaram as doses e os Níveis Sonoros Equivalentes a que os motoristas estariam expostos, os sintomas objetivos e subjetivos da perda auditiva e os efeitos combinados do ruído e vibração ou produtos químicos (monóxido de carbono) sobre sua audição. A avaliação audiométrica não deveria indicar somente a prevalência anual de alterações auditivas, porém, a partir da implementação de um padrão rígido para a obtenção dos resultados, possibilitaria garantir um panorama sobre a incidência de problemas auditivos ao longo dos anos. Além disso, estes motoristas deveriam participar de um Programa de Prevenção de Perdas Auditivas (PPPA), o qual primordialmente promove ações para evitar o desencadeamento ou o agravamento de perdas auditivas, bem como, os efeitos extra-auditivos causados pela exposição ao ruído intenso ou aos outros agentes de risco à audição ${ }^{2-7}$.

A qualidade de vida é um dos pilares das políticas sociais das empresas, em uma busca constante pelo equilíbrio psíquico, físico e social dos sujeitos dentro da organização ${ }^{8-10}$. A excelência dos produtos e serviços, assim como, suas inovações contínuas, somente se tornam possíveis se cada funcionário desfrutar de boa saúde, condições de trabalho, incentivos, turnos de trabalho regulares e jornada de trabalho adequada. O ruído acarreta alterações auditivas, extra-auditivas, além de problemas na saúde física e mental, tais como: distúrbios de sono, transtorno cardiovascular, uso de álcool e drogas, estresse, fadiga e tensão psicológica ${ }^{11-16}$. Conseqüentemente, as colisões envolvendo caminhões e os afastamentos dos motoristas por auxílio doença ou por acidente de trabalho seriam, muitas vezes, decorrentes das escassas ações de proteção à saúde desta categoria profissional.

A prática fonoaudiológica no contexto da Audiologia não tem se restringido à aplicação de uma bateria de exames audiométricos, mas tem se voltado à escuta do sujeito acerca dos problemas decorrentes da perda auditiva. Há uma escassez de trabalhos científicos sobre os efeitos do ruído na audição e na qualidade de vida em motoristas de caminhão.

Este trabalho teve como objetivo geral estudar a audição e a sua relação com a qualidade de vida em motoristas de caminhão.

Os objetivos específicos deste estudo pretenderam identificar a freqüência de alterações auditivas, bem como, verificar a relação entre os resultados da audiometria tonal liminar e do questionário sobre qualidade de vida na população estudada.

\section{MÉTODOS}

Realizou-se um estudo epidemiológico do tipo transversal de inquérito, a partir da avaliação audiométrica e do protocolo The World Health Organization Quality of Life Assessment Instrument Briefly - WHOQOL-bref ${ }^{17}$, aplicados nos motoristas de caminhão de uma distribuidora internacional (Figura 1).

Selecionou-se um dos clientes desta distribuidora para a realização da pesquisa. Seus motoristas de caminhão atuavam na distribuição de bebidas dentro do Estado de São Paulo. Os 75 motoristas de caminhão estudados eram do sexo masculino, com idades entre 27 a 61 anos e com tempo de profissão variando entre cinco a 40 anos, cuja seleção obedeceu aos seguintes critérios: trabalho atual exclusivamente como motorista de caminhão, tempo mínimo de cinco anos na atividade e carga horária média diária igual ou superior a oito horas.

Os valores das doses de ruído diárias, apresentados no Programa de Prevenção de Riscos Ambientais (PPRA) do ano de 2006 do cliente estudado, referentes aos modelos de caminhões Scania 124, Potência 330, Scania 124 Potência 420 e Iveco Frota 6501, podem ser encontrados na Tabela 1.

O dosímetro utilizado em ambos os PPRA era da marca Audio Dosímetro Digital, Modelo DOS 500 , fabricado pela Instrutherme.

Conforme demonstrado na Tabela 1, a exposição ao ruído era medida por meio de dose (em \% e em dB (A). Analisou-se os valores referentes a esta exposição, de acordo com os Limites de Tolerância, propostos pela Norma Regulamentadora (NR) $15-$ Atividades e Operações Insalubres, Anexo I (85 dB (A) por oito horas) ${ }^{18}$ e pelo preconizado na NR $9-$ Programa de Prevenção de Riscos Ambientais PPRA ${ }^{19}$.

A coleta dos dados foi efetuada no próprio local de trabalho dos sujeitos. A pesquisa iniciou-se no cliente (Jundiaí - SP) e posteriormente, em uma das filiais da distribuidora (São Paulo - Jaraguá SP). Primeiramente, realizou-se um contato com os controladores de tráfego, pois estes profissionais 


\section{WHOQOL - ABREVIADO - Versão em Português}

\section{A pontuação dos escores deverá ser realizada utilizando o programa estatístico SPSS, com a sintaxe do WHOQOL-bref.}

\begin{tabular}{|c|c|c|c|c|c|}
\hline \multicolumn{6}{|c|}{ Instruções } \\
\hline \multirow{2}{*}{\multicolumn{6}{|c|}{$\begin{array}{l}\text { Este questionário é sobre como você se sente a respeito de sua qualidade de vida, saúde e outras áreas de sua vida. Por favor } \\
\text { responda a todas as questões. Se você não tem certeza sobre que resposta dar em uma questão, por favor, escolha entre as } \\
\text { alternativas a que lhe parece mais apropriada. Esta, muitas vezes, poderá ser sua primeira escolha. } \\
\text { Por favor, tenha em mente seus valores, aspirações, prazeres e preocupações. Nós estamos perguntando o que você acha de } \\
\text { sua vida, tomando como referência as duas últimas semanas. Por exemplo, pensando nas últimas duas semanas, uma questão } \\
\text { poderia ser: }\end{array}$}} \\
\hline & & & & & \\
\hline & nada & muito pouco & médio & muito & completamente \\
\hline $\begin{array}{l}\text { Você recebe dos outros o } \\
\text { apoio de que necessita? }\end{array}$ & 1 & 2 & 3 & 4 & 5 \\
\hline
\end{tabular}

Você deve circular o número que melhor corresponde ao quanto você recebe dos outros o apoio de que necessita nestas últimas duas semanas.

Portanto, você deve circular o número 4 se você recebeu "muito" apoio como abaixo.

\begin{tabular}{|l|c|c|c|c|c|}
\hline & nada & muito pouco & médio & muito & completamente \\
\hline $\begin{array}{l}\text { Você recebe dos outros o } \\
\text { apoio de que necessita? }\end{array}$ & 1 & 2 & 3 & 4 & 5 \\
\hline
\end{tabular}

Você deve circular o número 1 se você não recebeu "nada" de apoio.

Por favor, leia cada questão, veja o que você acha e circule no número que lhe pareça ser a melhor resposta.

\begin{tabular}{|l|l|c|c|c|c|c|}
\hline & & muito ruim & ruim & $\begin{array}{c}\text { nem ruim } \\
\text { nem boa }\end{array}$ & boa & muito boa \\
\hline 1 & $\begin{array}{l}\text { Como você avaliaria sua } \\
\text { qualidade de vida? }\end{array}$ & 1 & 2 & 3 & 4 \\
\hline
\end{tabular}

As questões seguintes são sobre o quanto você tem sentido algumas coisas nas últimas duas semanas.

\begin{tabular}{|c|l|c|c|c|c|c|}
\hline & nada & muito pouco & mais ou menos & bastante & extremamente \\
\hline 3 & $\begin{array}{l}\text { Em que medida você acha } \\
\text { que sua dor (física) impede } \\
\text { você de fazer o que você } \\
\text { precisa? }\end{array}$ & 1 & 2 & 3 & 4 & 5 \\
\hline 4 & $\begin{array}{l}\text { O quanto você precisa de } \\
\text { algum tratamento médico } \\
\text { para levar sua vida diária? }\end{array}$ & 1 & 2 & 3 & 4 & 5 \\
\hline 5 & $\begin{array}{l}\text { O quanto você aproveita a } \\
\text { vida? }\end{array}$ & 1 & 2 & 3 & 4 & 5 \\
\hline 6 & $\begin{array}{l}\text { Em que medida você acha } \\
\text { que a sua vida tem sentido? }\end{array}$ & 1 & 2 & 3 & 4 & 5 \\
\hline 7 & $\begin{array}{l}\text { O quanto você consegue se } \\
\text { concentrar? }\end{array}$ & 1 & 2 & 3 & 4 & 5 \\
\hline 8 & $\begin{array}{l}\text { Quão seguro(a) você se } \\
\text { sente em sua vida diária? }\end{array}$ & 1 & 2 & 3 & 4 & 5 \\
\hline 9 & $\begin{array}{l}\text { Quão saudável é o seu } \\
\text { ambiente físico (clima, } \\
\text { barulho, poluição, } \\
\text { atrativos)? }\end{array}$ & 1 & 2 & 3 & 4 & 5 \\
\hline
\end{tabular}


As questões seguintes perguntam sobre quão completamente você tem sentido ou é capaz de fazer certas coisas nestas últimas duas semanas.

\begin{tabular}{|l|l|c|c|c|c|c|}
\hline & nada & muito pouco & médio & muito & completamente \\
\hline 10 & 1 & 2 & 3 & 4 & 5 \\
\hline 11 & $\begin{array}{l}\text { Você tem energia suficiente } \\
\text { para seu dia-a-dia? }\end{array}$ & $\begin{array}{l}\text { Você é capaz de aceitar sua } \\
\text { aparência física? }\end{array}$ & 1 & 2 & 3 & 4 \\
\hline 12 & $\begin{array}{l}\text { Você tem dinheiro suficiente } \\
\text { para satisfazer suas } \\
\text { necessidades? }\end{array}$ & 1 & 2 & 3 & 4 & 5 \\
\hline 13 & $\begin{array}{l}\text { Quão disponíveis para você } \\
\text { estão as informações que } \\
\text { precisa no seu dia-a-dia? }\end{array}$ & 1 & 2 & 3 & 4 & 5 \\
\hline 14 & $\begin{array}{l}\text { Em que medida você tem } \\
\text { oportunidades de atividade } \\
\text { de lazer? }\end{array}$ & 1 & 2 & 3 & 4 & 5 \\
\hline
\end{tabular}

As questões seguintes perguntam sobre quão bem ou satisfeito você se sentiu a respeito de vários aspectos de sua vida nas últimas duas semanas.

\begin{tabular}{|c|c|c|c|c|c|c|}
\hline & & muito ruim & ruim & $\begin{array}{l}\text { nem ruim } \\
\text { nem bom }\end{array}$ & bom & muito bom \\
\hline \multirow[t]{2}{*}{15} & $\begin{array}{l}\text { Quão bem você é capaz de } \\
\text { se locomover? }\end{array}$ & 1 & 2 & 3 & 4 & 5 \\
\hline & & muito insatisfeito & Insatisfeito & $\begin{array}{l}\text { nem satisfeito } \\
\text { nem insatisfeito }\end{array}$ & satisfeito & muito satisfeito \\
\hline 16 & $\begin{array}{l}\text { Quão satisfeito(a) você está } \\
\text { com o seu sono? }\end{array}$ & 1 & 2 & 3 & 4 & 5 \\
\hline 17 & $\begin{array}{l}\text { Quão satisfeito(a) você está } \\
\text { com sua capacidade de } \\
\text { desempenhar as atividades } \\
\text { do seu dia-a-dia? }\end{array}$ & 1 & 2 & 3 & 4 & 5 \\
\hline 18 & $\begin{array}{l}\text { Quão satisfeito(a) você está } \\
\text { com sua capacidade para o } \\
\text { trabalho? }\end{array}$ & 1 & 2 & 3 & 4 & 5 \\
\hline 19 & $\begin{array}{l}\text { Quão satisfeito(a) você está } \\
\text { consigo mesmo? }\end{array}$ & 1 & 2 & 3 & 4 & 5 \\
\hline 20 & $\begin{array}{l}\text { Quão satisfeito(a) você está } \\
\text { com suas relações pessoais } \\
\text { (amigos, parentes, } \\
\text { conhecidos, colegas)? }\end{array}$ & 1 & 2 & 3 & 4 & 5 \\
\hline 21 & $\begin{array}{l}\text { Quão satisfeito(a) você está } \\
\text { com sua vida sexual? }\end{array}$ & 1 & 2 & 3 & 4 & 5 \\
\hline 22 & $\begin{array}{l}\text { Quão satisfeito(a) você está } \\
\text { com o apoio que você } \\
\text { recebe de seus amigos? }\end{array}$ & 1 & 2 & 3 & 4 & 5 \\
\hline 23 & $\begin{array}{l}\text { Quão satisfeito(a) você está } \\
\text { com as condições do local } \\
\text { onde mora? }\end{array}$ & 1 & 2 & 3 & 4 & 5 \\
\hline 24 & $\begin{array}{l}\text { Quão satisfeito(a) você está } \\
\text { com o seu acesso aos } \\
\text { serviços de saúde? }\end{array}$ & 1 & 2 & 3 & 4 & 5 \\
\hline 25 & $\begin{array}{l}\text { Quão satisfeito(a) você está } \\
\text { com o seu meio de } \\
\text { transporte? }\end{array}$ & 1 & 2 & 3 & 4 & 5 \\
\hline
\end{tabular}


As questões seguintes referem-se a com que freqüência você sentiu ou experimentou certas coisas nas últimas duas semanas.

\begin{tabular}{|l|l|c|c|c|c|c|}
\hline & nunca & algumas vezes & freqüentemente & $\begin{array}{c}\text { muito } \\
\text { freqüentemente }\end{array}$ & sempre \\
\hline 26 & $\begin{array}{l}\text { Com que freqüência você } \\
\text { tem sentimentos negativos } \\
\text { tais como: mau humor, } \\
\text { desespero, ansiedade e } \\
\text { depressão? }\end{array}$ & 1 & 2 & 3 & 4 & 5 \\
\hline
\end{tabular}

Alguém Ihe ajudou a preencher este questionário?

Quanto tempo você levou para preencher este questionário?

Você tem algum comentário sobre o questionário?

OBRIGADA PELA SUA COLABORAÇÃO!

Figura 1 - Questionário WHOQOL

Tabela 1 - Avaliação do nível de exposição diária ao ruído pelos motoristas de caminhão, constatada em 2006 no PPRA

\begin{tabular}{lcc}
\hline Modelo do Caminhão & $\begin{array}{c}\text { Leq } \\
(\mathrm{em} \mathrm{dB}(\mathrm{A}))\end{array}$ & $\begin{array}{c}\text { Dose de ruído } \\
\text { (em \%) }\end{array}$ \\
\hline $\begin{array}{l}\text { Scania 124 Potência 330 } \\
\text { Scania 124 Potência 420 } \\
\text { Iveco Frota 6501 }\end{array}$ & 81,9 & $65 \%$ \\
\hline
\end{tabular}

Legenda:

PPRA - Programa de Prevenção de Riscos Ambientais

Leq - Nível Sonoro Equivalente

$\mathrm{dB}(\mathrm{A})$ - Decibel Nível de Audição

relacionavam-se diretamente com os motoristas de caminhão, nos horários em que iniciavam suas jornadas de trabalho. Tal medida sobre este primeiro contato era adotada, pois a fonoaudióloga não poderia estar sempre presente devido à distância entre as cidades e também pelas diferentes escalas de trabalho existentes. Portanto, explicou-se o objetivo e o procedimento da pesquisa por meio de contato telefônico e carta informativa, enviada por e-mail.

Solicitou-se aos controladores de tráfego que convidassem todos os motoristas de caminhão a participarem da pesquisa e, após repassarem as informações, agendavam os dias e horários em que a fonoaudióloga estaria na distribuidora. Os controladores salientavam que a colaboração deveria ser voluntária, não havendo prejuízo em relação ao seu emprego. Nos respectivos dias e horários, solicitouse aos motoristas que chegassem duas horas antes do início de seu expediente, para preencherem todos os instrumentos da pesquisa, caso desejassem participar. Novamente, houve a explicação do objetivo e do procedimento da pesquisa de forma oral, cabendo aos sujeitos decidirem sobre a sua participação.

O procedimento da pesquisa consistia no preenchimento da anamnese pela fonoaudióloga junto ao motorista de caminhão, na avaliação audiométrica e no preenchimento do WHOQOL-bref. Todo o procedimento realizou-se em um único dia, para que fosse garantida a atenção ao sujeito individualmente e o repouso auditivo necessário à avaliação audiométrica (14 horas). As audiometrias tonais eram efetuadas em cabina acústica móvel, da marca Acústica São Luiz, calibrada de acordo com a American National Standards Institute (ANSI) S3: $1^{20}$, deslocada para os locais selecionados. Previamente à realização da audiometria tonal, realizou-se a inspeção visual do meato acústico externo, a fim de se verificar alguma impossibilidade para a execução do exame, como cerume parcial ou total. Para tanto, o otoscópio utilizado era o modelo Heine mini 2000. 
Posteriormente, realizou-se a audiometria tonal liminar, tanto por via aérea, nas freqüências de 0.25 $\mathrm{kHz}, 0.5 \mathrm{kHz}, 1 \mathrm{kHz}, 2 \mathrm{kHz}, 3 \mathrm{kHz}, 4 \mathrm{kHz}, 6 \mathrm{kHz}$ e $8 \mathrm{kHz}$, quanto por via óssea, se necessário. $\mathrm{Na}$ logoaudiometria, adotou-se a pesquisa do Limiar de Reconhecimento de Fala (LRF) e do Índice de Reconhecimento de Fala (IRF). Para a execução destes exames, o audiômetro utilizado era da marca Maico, modelo MA41, calibrado anualmente ${ }^{21,22}$. O resultado da audiometria tonal foi explicado a cada motorista de caminhão.

A primeira questão do protocolo WHOQOL-bref era lida e efetuada oralmente pela examinadora, a fim de garantir a inteligibilidade das demais pelo entrevistado. A resposta era anotada pelo próprio sujeito em seu questionário, assinalando a resposta que melhor the conviesse. As seguintes questões eram auto-administradas pelo sujeito, sob a supervisão da fonoaudióloga. Cada questão tinha cinco alternativas como resposta, cabendo ao sujeito assinalar uma como sua melhor opção. No caso de dúvida, a examinadora releu a questão de forma pausada ao sujeito, evitando dar sinônimos às palavras das perguntas. Enfatizou-se que todo o questionário referia-se às duas últimas semanas, independente do local em que os sujeitos se encontravam. Cada motorista de caminhão dispensou, em média, uma hora para realizar todas as etapas propostas pela pesquisa.

As avaliações audiométricas foram divididas em três Grupos, de acordo com os resultados das oreIhas conjuntamente ${ }^{23}$ :

Grupo 1 (audiogramas sugestivos de audição dentro dos padrões de normalidade): sujeitos que apresentaram todos os limiares obtidos bilateralmente em valores iguais ou menores a $25 \mathrm{dBNA}$.

Grupo 2 (audiogramas sugestivos de Perda Auditiva Induzida por Ruído - PAIR): sujeitos que apresentaram configuração de perda audiométrica (limiares maiores que $25 \mathrm{dBNA}$ ) nas altas freqüências ( $3 \mathrm{kHz}$ e/ou $4 \mathrm{kHz}$ e/ou $6 \mathrm{kHz}$ ).

Grupo 3 (audiogramas com outras classificações): sujeitos que apresentaram perda auditiva, cuja configuração audiométrica não foi compatível com os Grupos 1 e 2.

Desta forma, o Grupo Normalidade subdividiuse em:

- Normalidade bilateral: Todos os limiares auditivos, tanto na orelha direita quanto na orelha esquerda, eram iguais ou inferiores a $25 \mathrm{~dB}$ NA;

- Normalidade com entalhe unilateral: Idem ao descrito em normalidade bilateral, porém apresentando entalhe audiométrico em apenas uma orelha (rebaixamento dentro da normalidade nas freqüências de $3 \mathrm{kHz}, 4 \mathrm{kHz}$ ou $6 \mathrm{kHz}$, sendo con- siderado quando havia diferença de pelo menos 10 dBNA da freqüência anterior ou posterior);

- Normalidade com entalhe bilateral: Idem ao descrito em normalidade bilateral, porém apresentando entalhe audiométrico em ambas as orelhas.

O Grupo Sugestivo de PAIR subdividiu-se em:

- PAIR bilateral: traçado audiométrico sugestivo de PAIR em ambas as orelhas;

- PAIR unilateral: uma orelha com traçado audiométrico sugestivo de PAIR, com a outra apresentando os limiares dentro dos padrões de normalidade (iguais ou inferiores a $25 \mathrm{~dB} \mathrm{NA}$ );

- PAIR unilateral com entalhe na outra oreIha: traçado audiométrico sugestivo de PAIR em uma orelha e traçado dentro dos padrões de normalidade com entalhe audiométrico na outra orelha.

O Grupo Outros apresentou traçados audiométricos sugestivos de perda descendente (maior rebaixamento em $8 \mathrm{kHz}$ ), bilateralmente ou com uma das orelhas dentro dos padrões de normalidade.

O WHOQOL-bref é um questionário baseado no WHOQOL-100, o qual é composto por 26 questões, com escalas de respostas que correspondem a: intensidade (de nada a extremamente), capacidade (de nada a completamente), freqüência (de nunca a sempre) e avaliação (de muito insatisfeito a muito satisfeito, de ruim a muito bom). Este protocolo está dividido em quatro domínios: Físico, Psicológico, Relações Sociais e Meio Ambiente. A análise dos escores do WHOQOL-bref foi realizada seguindo as fórmulas originais para a computação das respostas, elaborado por Alison Harper e Mick Power para o Grupo de Qualidade de Vida da Organização Mundial de Saúde ${ }^{17}$. As versões em Português dos instrumentos WHOQOL-100 e WHOQOL-bref foram desenvolvidas no "Centro WHOQOL para o Brasil“", no Departamento de Psiquiatria e Medicina Legal da Universidade Federal do Rio Grande do Sul ${ }^{24}$.

A pesquisa foi aprovada pelo Comitê de Ética do Programa de Estudos Pós-Graduados em Fonoaudiologia da Pontifícia Universidade Católica de São Paulo (PEPG em Fonoaudiologia - PUCSP), sob parecer de número 0002/2005. Um Termo de Consentimento Livre e Esclarecido foi elaborado, sendo lido e devidamente assinado pelos sujeitos antes do atendimento.

Um banco de dados foi criado para a verificação da consistência das respostas, para posteriormente, ser procedida à análise estatística.

Foram utilizados basicamente testes não paramétricos, pois as condições mínimas para a utilização de testes paramétricos, como a normalidade da distribuição e a homocedasticidade (homogeneidade das variâncias), não foram consideradas satisfatórias. Portanto, os testes Qui-Quadrado 
para Independência e Correlação de Spearman foram aplicados na análise estatística das variáveis selecionadas.

O nível de significância proposto foi de $5 \%$ $(p<0,05)$, conforme sugerido para estudos biológicos. Todos os intervalos de confiança foram construídos com $95 \%$ de confiança estatística.

\section{RESULTADOS}

\section{Análise da Avaliação Audiométrica}

Em relação aos 75 audiogramas estudados, 50 sujeitos $(66,7 \%)$ apresentaram audiogramas dentro dos padrões de normalidade (Grupo 1) e 25 sujeitos $(33,3 \%)$ apresentaram audiogramas com alterações (Grupo 2 e 3).

Dentre os 50 audiogramas do Grupo $1(100,0 \%)$, $14(28,0 \%)$ classificaram-se como dentro dos padrões de normalidade, com entalhe unilateral, $31(62,0 \%)$ eram classificados como dentro dos padrões de normalidade, com entalhe bilateral e cinco $(10,0 \%)$ apresentaram audiogramas dentro dos padrões de normalidade, sem entalhe.

Dos 25 audiogramas alterados $(100,0 \%), 20$ $(80,0 \%)$ classificaram-se como sugestivos de PAIR (Grupo 2) e cinco $(20,0 \%)$ eram sugestivos de outras causas (Grupo 3). Dentre os 20 audiogramas do Grupo $2(100,0 \%), 16(80,0 \%)$ classificaram-se sugestivos de PAIR bilateral e quatro $(20,0 \%)$ eram sugestivos de PAIR unilateral, sendo que as orelhas contralaterais apresentaram audição dentro dos padrões de normalidade, com entalhe. Dos audiogramas presentes no Grupo 3 - Outros (100,0\%), um sujeito $(20,0 \%)$ apresentou perda auditiva condutiva unilateral, um $(20,0 \%)$ apresentou perda auditiva mista unilateral e três $(60 \%)$ resultaram em perdas auditivas neurossensoriais, sendo duas $(66,7 \%)$ bilaterais e uma $(33,3 \%)$ unilateral.

Neste estudo, dos 75 (100\%) testados, utilizouse os dados de $70(93,3 \%)$ sujeitos. Foram excluídos os cinco sujeitos (6,7\%) pertencentes ao Grupo Outros, pois não apresentavam audiogramas classificados como sugestivos de PAIR. A Tabela 2 apresentou a divisão das avaliações audiométricas nos Grupos 1 e 2 propostos por Fiorini (1994), considerando o resultado da pior orelha para a classificação. Dos 70 (100\%) sujeitos analisados, 50 (71,4\%) apresentaram resultados dentro dos padrões de normalidade bilateralmente, pertencendo ao Grupo 1 - Normalidade e 20 (28,6\%) classificaram-se no Grupo 2 - Sugestivo de PAIR, em pelo menos uma orelha.

$\mathrm{Na}$ Tabela 3, verificou-se a existência de uma associação e/ou dependência entre os resultados obtidos na avaliação audiométrica, por meio da categorização utilizada por Fiorini (1994) (Grupos 1 e 2) e a variável qualitativa anos de profissão. Portanto, o sujeito com maior tempo de profissão como motorista de caminhão pertenceu ao Grupo 2 - Sugestivo de PAIR e aquele com menor tempo na carreira pertenceu ao Grupo 1 - Normalidade.

Tabela 2 - Distribuição do critério utilizado por Fiorini (1994), em porcentagem (\%), classificando em Grupos 1 e 2 os resultados da avaliação audiométrica dos motoristas de caminhão $(\mathrm{N}=70)$

\begin{tabular}{lcc}
\hline Grupos & Sujeitos (N) & $\%$ \\
\hline Grupo 1 - Normal & 50 & 71,4 \\
Grupo 2 - Sugestivo de PAIR & 20 & 28,6 \\
\hline Total & 70 & 100,0 \\
\hline
\end{tabular}

Tabela 3 - Distribuição da variável qualitativa: Anos de Profissão, considerando os resultados obtidos na avaliação audiométrica dos motoristas de caminhão, através da categorização nos Grupos utilizados por Fiorini (1994) ( $\mathrm{N}=70)$

\begin{tabular}{|c|c|c|c|c|c|c|}
\hline \multirow{3}{*}{ Grupos } & \multicolumn{4}{|c|}{ Anos de Profissão } & \multirow{2}{*}{\multicolumn{2}{|c|}{ Total }} \\
\hline & \multicolumn{2}{|c|}{$\leq 15$ anos } & \multicolumn{2}{|c|}{$>15$ anos } & & \\
\hline & $\mathbf{N}$ & $\%$ & $\mathbf{N}$ & $\%$ & $\mathbf{N}$ & $\%$ \\
\hline Grupo 1 & 28 & $56,0 \%{ }^{*}$ & 22 & $44,0 \%$ & 50 & $100,0 \%$ \\
\hline Grupo 2 & 6 & $30,0 \%$ & 14 & $70,0 \%{ }^{*}$ & 20 & $100,0 \%$ \\
\hline Total & 34 & $48,6 \%$ & 36 & $51,4 \%$ & 70 & $100,0 \%$ \\
\hline
\end{tabular}

Legenda:

* $p$-valor $=0,049$

Teste Qui-Quadrado para Independência 
Tabela 4 - Distribuição da variável qualitativa: Idade, considerando os resultados obtidos na avaliação audiométrica dos motoristas de caminhão, através da categorização nos Grupos utilizados por Fiorini (1994) ( $N=70)$

\begin{tabular}{|c|c|c|c|c|c|c|}
\hline \multirow{3}{*}{ Grupos } & \multicolumn{4}{|c|}{ Idade } & \multirow{2}{*}{\multicolumn{2}{|c|}{ Total }} \\
\hline & \multicolumn{2}{|c|}{$\leq 45$ anos } & \multicolumn{2}{|c|}{$>45$ anos } & & \\
\hline & $\mathbf{N}$ & $\%$ & $\mathbf{N}$ & $\%$ & $\mathbf{N}$ & $\%$ \\
\hline Grupo 1 & 37 & $74,0 \%{ }^{*}$ & 13 & $26,0 \%$ & 50 & $100,0 \%$ \\
\hline Grupo 2 & 8 & $40,0 \%$ & 12 & $60,0 \% *$ & 20 & $100,0 \%$ \\
\hline Total & 45 & $64,3 \%$ & 25 & $35,7 \%$ & 70 & $100,0 \%$ \\
\hline
\end{tabular}

Legenda:

${ }^{*} \mathrm{p}$-valor $=0,007$

Teste Qui-Quadrado para Independência

Na Tabela 4, observou-se a existência de uma associação e/ou dependência entre os resultados obtidos na avaliação audiométrica, por meio da categorização utilizada por Fiorini (1994) (Grupos 1 e 2) e a variável qualitativa idade. Portanto, o sujeito mais velho pertenceu ao Grupo 2 - Sugestivo de PAIR e o mais novo, ao Grupo 1 - Normalidade.

\section{Análise da Qualidade de vida}

As Tabelas 5 e 6 apresentaram a comparação dos domínios do protocolo WHOQOL-bref, considerando os resultados da avaliação audiométrica utilizada por Fiorini (1994) (Grupo 1 - Normalidade e Grupo 2 - Sugestivo de PAIR). Portanto, observouse que algumas correlações entre os domínios do protocolo WHOQOL-bref e os Grupos 1 e 2 empregados por Fiorini (1994) eram consideradas estatisticamente significantes.

\section{DISCUSSÃO}

Os motoristas de caminhão apresentaram audiogramas simétricos. Dentre os 50 audiogramas do Grupo 1 (71,4\%), 31 encontravam-se dentro dos padrões de normalidade, com entalhe bilateral $(62,0 \%)$ e dos 20 audiogramas do Grupo $2(28,6 \%), 16(80,0 \%)$ foram sugestivos de PAIR bilateral (Tabela 2). A combinação do ambiente reverberante e do contínuo movimento de cabeça do sujeito durante o trabalho resultariam em exposição semelhante para ambas as orelhas. Portanto, exposições ao ruído contínuo não produziriam assimetria, o que se verificou na maior parte dos audiogramas analisados ${ }^{6}$.

Outro fator inesperado e alarmante foi a prevalência de perdas auditivas sugestivas de PAIR $(28,6 \%)$ encontradas nesta classe profissional. Este dado seria equivalente ou superior ao encontrado em muitos estudos sobre o perfil audiométrico de trabalhadores de indústrias. Os trabalhadores da indústria metalúrgica apresentaram $23,7 \%$ dos audiogramas sugestivos de PAIR ${ }^{23}$. Esta prevalência também foi maior do que a encontrada em motoristas de caminhão: $11,5 \%$ de audiogramas classificaram-se como sugestivos de PAIR ${ }^{3}$. Por isso, a implantação de um Programa de Prevenção de Perdas Auditivas (PPPA) nas distribuidoras seria necessária, a fim de garantir o monitoramento auditivo, bem como, o treinamento sobre a saúde auditiva aos motoristas de caminhão.

No PPRA do ano de 2006 da Distribuidora, os valores obtidos quanto ao Leq e à dose estiveram abaixo dos Limites de Tolerância (85 dB (A) por oito horas) ${ }^{18}$. Porém, mesmo as medições do Leq e das doses não estando aquém do permitido, estas excederam os valores de exposição ao ruído, recomendados pela Legislação Brasileira, preconizado na NR 9 - Programa de Prevenção de Riscos Ambientais - PPRA ${ }^{19}$. Medições de ruído acima do Nível de Ação (80 dB(A) por oito horas) já seriam prejudiciais à saúde auditiva. Portanto, a partir destes valores obtidos já poderiam ser adotadas medidas preventivas em relação ao desencadeamento ou agravamento de alterações auditivas.

A Tabela 3 revelou que existiu uma associação e/ou dependência estatística entre os resultados audiométricos presentes no Grupo 1 e 2 e os anos de profissão $(p=0,049)$. Com isso, 28 motoristas $(56,0 \%)$ com tempo de profissão inferior a 15 anos apresentaram audiogramas dentro dos padrões de normalidade, porém, 14 sujeitos $(70,0 \%)$ com anos de profissão superiores a 15 anos apresentaram alterações auditivas sugestivas de PAIR. Portanto, estes dados corroboraram com a literatura, já que a exposição dos motoristas de caminhão ao Nível de Pressão Sonora (NPS) elevado ao longo dos anos se caracterizaria como um risco potencial para o agravamento ou desencadeamento da PAIR ${ }^{4}$.

$\mathrm{Na}$ Tabela 4, a variável qualitativa idade era estatisticamente significante, quando comparada aos resultados audiométricos presentes no 
Grupo 1 e $2(p=0,007)$. Com isso, o motorista de caminhão que apresentou audiograma com alteração auditiva era mais velho em relação aos que demonstraram audiogramas dentro dos padrões de normalidade. Observou-se que 12 sujeitos (60,0\%) apresentaram idades superiores a 45 anos e perda auditiva sugestiva de PAIR. Em contrapartida, verificou-se que 37 sujeitos $(74,0 \%)$ apresentaram idades inferiores a 45 anos e audição dentro dos padrões de normalidade. Os dados concordaram com a literatura porque, além da suscetibilidade auditiva individual ${ }^{6}$, os motoristas de caminhão poderiam apresentar perdas auditivas em decorrência da idade, associada à exposição constante ao NPS elevado durante sua jornada de trabalho, decorrente de suas condições, turnos e cargas de trabalho ${ }^{5}$.

Os achados das Tabelas 3 e 4 apontaram que, quanto maior a idade e o tempo de profissão como motorista de caminhão, tanto maior é a sua alteração auditiva, principalmente devido à exposição ao ruído elevado durante jornada de trabalho. Isto demonstrou o quanto o ruído intenso no ambiente de trabalho pode ser prejudicial para a saúde auditiva dos motoristas de caminhão ${ }^{2}$. Portanto, o elevado Nível de Pressão Sonora associado aos anos de profissão e/ou à idade podem comprometer a audição e o bem-estar (físico e emocional) dos motoristas de caminhão ${ }^{1,4-7,11-16}$.

As Tabelas 5 e 6 verificaram algumas correlações estatisticamente significantes nos Grupos $1 \mathrm{e}$ 2 em relação aos domínios do WHOQOL-bref. No Grupo 1, as correlações eram: Físico x Psicológico $(p<0,001)$, Social x Físico $(p<0,001)$, Meio Ambiente $x$ Físico $(p<0,001)$, Psicológico $x$ Social $(p=0,002)$, Psicológico $x$ Meio Ambiente $(p=0,001)$ e Meio Ambiente $x$ Social $(p<0,001)$. No Grupo 2, as correlações eram: Físico x Psicológico $(p=0,002)$, Psicológico $\times$ Social $(p<0,001)$, Psicológico $\times$ Ambiente $(p=0,005)$ e Ambiente $\times$ Social $(p=0,026)$. Os achados discordaram da literatura, pois a qualidade de vida não esteve relacionada diretamente aos resultados da avaliação audiológica, porque inde-

Tabela 5 - Distribuição dos domínios do protocolo WHOQOL-bref, considerando os resultados da avaliação audiométrica do Grupo 1 - Normalidade, realizada nos motoristas de caminhão $(\mathrm{N}=70)$

\begin{tabular}{cccccc}
\hline \multicolumn{2}{c}{ Grupo 1 } & Físico & Psicológico & Social & $\begin{array}{c}\text { Meio } \\
\text { Ambiente }\end{array}$ \\
\hline Psicológico & Correlação & $67,8 \%$ & - & - & - \\
& p-valor & $<0,001^{*}$ & - & - & - \\
Social & Correlação & $63,6 \%$ & $43,6 \%$ & - & - \\
& p-valor & $<0,001^{*}$ & $0,002^{*}$ & - & - \\
Meio & Correlação & $70,8 \%$ & $47,0 \%$ & $48,3 \%$ & - \\
Ambiente & p-valor & $<0,001^{*}$ & $0,001^{*}$ & $<0,001^{*}$ & - \\
& &
\end{tabular}

Legenda:

${ }^{*} p<0,05$

Teste de Correlação de Spearman

Tabela 6 - Distribuição dos domínios do protocolo WHOQOL-bref, considerando os resultados da avaliação audiométrica do Grupo 2 (Sugestivo de PAIR), realizada nos motoristas de caminhão $(\mathrm{N}=70)$

\begin{tabular}{cccccc}
\hline \multicolumn{2}{c}{ Grupo 2} & Físico & Psicológico & Social & $\begin{array}{c}\text { Meio } \\
\text { Ambiente }\end{array}$ \\
\hline Psicológico & Correlação & $64,5 \%$ & - & - & - \\
& p-valor & $0,002^{*}$ & - & - & - \\
Social & Correlação & $52,6 \%$ & $71,1 \%$ & - & - \\
& p-valor & $0,017^{*}$ & $<0,001^{*}$ & - & - \\
Meio & Correlação & $34,6 \%$ & $60,4 \%$ & $49,5 \%$ & - \\
Ambiente & p-valor & 0,135 & $0,005^{\star}$ & $0,026^{*}$ & - \\
\hline
\end{tabular}

Legenda:

${ }^{*} \mathrm{p}<0,05$

Teste de Correlação de Spearman 
pendentemente dos resultados dos audiogramas, os domínios do WHOQOL- bref eram significantes 17,24. O termo qualidade de vida incluiria uma variedade potencial de condições que poderiam afetar a percepção do sujeito, seus sentimentos e comportamentos relacionados ao seu funcionamento diário, incluindo, mas não se limitando, a sua condição de saúde. Portanto, a qualidade de vida estaria ligada ao impacto do estado de saúde sobre a capacidade do sujeito de viver plenamente ${ }^{8-10}$.

Por intermédio do questionário WHOQOL-bref, a qualidade de vida nos motoristas de caminhão apresentou-se para a maioria como "Boa" (44 sujeitos $-58,7 \%$ ) ou "Muito Boa" (18 sujeitos $-24,0 \%$ ). Porém, este conceito refletiu o cotidiano das duas últimas semanas vividas por eles, de acordo com as instruções de preenchimento seguidas pelo protocolo. Por isso, por meio do questionário adotado, a qualidade de vida poderia estar adequada no presente momento, porém futuramente, poderá ser comprometida. O dado, portanto, torna-se pontual. A qualidade de vida deveria ser precedida da discussão sobre condições de vida, a fim de se garantir a qualidade de moradia, educação, saneamento básico e alimentação na vida do sujeito ${ }^{8-10}$.

\section{CONCLUSÂO}

Diante dos resultados do presente estudo que teve como objetivo geral estudar a audição e a qualidade de vida em motoristas de caminhão foi possível concluir que a freqüência de alterações auditivas na população estudada era de $28,6 \%$. Os anos de profissão e a idade influenciaram estatisticamente os resultados da audiometria tonal. Contudo, a qualidade de vida não esteve relacionada aos resultados obtidos na audiometria tonal.

\begin{abstract}
Purpose: to study hearing in relation to the quality of life in truck drivers. Methods: an inquiring transversal epidemiological study was realized in 75 truck drivers, who answered to anamnesis and filled the World Health Organization questionnaire on quality of life - WHOQOL-bref, and underwent pure tone and speech audiometry. Noise induced hearing loss (NIHL) was classified, according to Fiorini's (1994) criterion. Results: out of 75 evaluated audiograms, 50 subjects $(66.7 \%)$ showed normal hearing (Group 1) and 25 subjects (33.3\%) showed altered audiograms, being 20 (80.0\%) classified as suggestive of $\mathrm{NIHL}$ (Group 2 ) and $5(20,0 \%)$ were suggestive of other causes (Group 3 ). There was an association and/or dependence between groups and age $(p=0,007)$ and between groups and years in the profession $(p=0.049)$. In Group 1 - Normal, there were statistically significant correlations for the domains: Physical $x$ Psychological $(p<0.001)$, Social $x$ Physical $(p<0.001)$, Environment $x$ Physical $(p<0.001)$, Psychological $\times$ Social $(p=0,002)$, Psychological $x$ Environment $(p=0,001)$ and Environment $x$ Social $(p<0.001)$. In the Group $2-$ Suggestive of NIHL, the correlations were: Physical $x$ Psychological $(p=0.002)$, Psychological $x$ Social $(p<0.001)$, Psychological $x$ Environment $(p=0.005)$ and Environment $x$ Social $p=(0.026)$. Conclusion: the prevalence of hearing disorders, suggesting NIHL, was $28.6 \%$ in this professional category, but the quality of life was not related to the audiogram results.
\end{abstract}

KEYWORDS: Hearing Loss; Quality of Life; Occupational Health; Noise, Occupational; Audiometry; Audiology

\section{REFERÊNCIAS}

1. Sarra CA, Vilela JC, Oliveira SM. Aspectos relacionados à vida social, convivência familiar e dificuldades no trabalho dos caminhoneiros [monografia]. São Paulo (SP): Faculdade de Medicina da Universidade de São Paulo; 1998.

2. Seshagiri B. Occupational noise exposure of operators of heavy trucks. Am Ind Hyg Assoc J. 1998; 59(3):205-13.

3. Cepinho CP, Corrêa A, Bernardi APA. Ocorrência de perda auditiva em motoristas de ônibus e caminhões de São Paulo. Rev CEFAC. 2003; 5(2):181-6.

4. Van den Heever DJ, Roets FJ. Noise exposure of truck drivers: a comparative study. Am Ind Hyg Assoc J. 1996; 57(6):564-6.

5. Koda S, Yasuda N, Sugihara $Y$, Ohara H, Udo H, Otani T, et al. Analyses of work-relatedness of health problems among truck drivers by questionnaire survey. Sangyo Eiseigaku Zasshi. 2000; 42(1):6-16.

6. Clark WW. Five myths in assessing the effects of noise on hearing. Central Institute for the Deaf [periódico on line]. Disponível em: URL: http://www.heal- 
thyhearing.com/library/article_content.asp?article_ id=42 Acesso em: 24 jan 2006.

7. Lacerda A, Leroux T, Gagné JP. The combined effect of noise and carbon monoxide on hearing thresholds of exposed workers. J Acoust Soc Am. 2005; 117(4):2481.

8. Bullinger M, Anderson R, Cella D, Aaronson N. Developing and a evaluating cross-cultural instruments from minimum requirements to optimal models. Qual Life Res. 1993; 2(6):451-9.

9. Lapcevic M, Demitrijevic I, Ristic J, Vukovic M. Rating of life valuation scale. Srp Arh Celok Lek. 2006; 134(9-10):432-7.

10. Bullinger M. Methodological basis and aspects of quality of life. Dtsch Med Wochenschr. 2006; 131(19 suppl 1):S5-7.

11. Brown CJ. Wake-up call issued about drowsy truck drivers. Can Med Assoc J. 1997; 157(9):1195. 12. Leigh JP, Miller TR. Job-related diseases and occupations within a large workers' compensation data set. Am J Ind Med. 1998; 33(3):197-211.

13. De Croon EM, Blonk RW, Van der Beek J, FringsDresen $\mathrm{MH}$. The trucker strain monitor: an occupation-specific questionnaire measuring psychological job strain. Int Arch Occup Environ Health. 2001; 74(6):429-36.

14. Drummer $\mathrm{OH}$, Gerostamoulos J, Batziris $\mathrm{H}$, Chu M, Caplehorn J, Robertson MD, et al. The involvement of drugs in drivers of motor vehicles killed in australian road traffic crashes. Accid Anal Prev. 2004; 36(2):239-48.

15. Solomon AJ, Doucette JT, Garland E, McGinn T. Healthcare and the long haul: long distance truck drivers a medically underserved population. Am J Ind Med. 2004; 46(5):463-71.

16. Hartley LR, El Hassani J. Stress, violations and accidents. Appl Ergon. 1994; 25(4):221-30.
17. The WHOQOL Group. Development of the World Health Organization WHOQOL-BREF quality of life assessment. Psychol Med. 1998; 28(3):551-8.

18. Brasil. Ministério do Trabalho. Norma Regulamentadora 15. Atividades e operações insalubres. In: Segurança e Medicina do Trabalho Lei $n^{\circ} 6.514$ de 22/12/1977. 53. ed. São Paulo: Atlas; 2003. p. $133-214$.

19. Brasil. Ministério do Trabalho. Norma Regulamentadora 9. Programa de Prevenção de Riscos Ambientais - PPRA. In: Segurança e Medicina do Trabalho Lei $n^{\circ} 6.514$ de 22/12/1977. 53. ed. São Paulo: Atlas; 2003. p. 101-5.

20. American National Standards Institute. Maximum permissible ambient noise levels for audiometric testing rooms - ANSI, S3:1, New York; 1991.

21. International Organization for Standardization (ISO). Acoustics audiometric test methods - Part 1: basic pure tone air and bone conduction threshold audiometry: ISO 8253-1. Genève, Switzerland; 1989.

22. Momensohn-Santos TMM, Russo ICP, Assayag FM, Lopes LQ. Determinação dos limiares tonais por via aérea e por via óssea. In: Momensohn-Santos TMM, Russo ICP, organizadoras. Prática da audiologia clínica. 4. ed. São Paulo: Cortez; 2005. p. 67-96.

23. Fiorini AC. Conservação auditiva: estudo sobre o monitoramento audiométrico em trabalhadores de uma indústria metalúrgica [mestrado]. São Paulo (SP): Pontifícia Universidade Católica de São Paulo; 1994.

24. Fleck MPA, Leal OF, Louzada S, Xavier M, Chachamovich E, Vieira G, et al. O instrumento de avaliação de qualidade de vida abreviado da Organização Mundial de Saúde (WHOQOL-bref): aplicação da versão em português. Rev Saúde Pública. 2000; 34:178-83.

RECEBIDO EM: 03/07/2007

ACEITO EM: 10/09/2007

Endereço para correspondência:

Rua Gervásio Pires Ferreira, 120

São Paulo - SP

CEP: 04072-050

Tel: (11) 22753833 / 81222121

Fax: (11) 22753833

E-mail: gabilopes21@terra.com.br 